\title{
Selecting Mobile Office Devices using a Goal-Oriented Approach
}

\author{
Carlos Cares \\ Depto. Ingeniería de Sistemas \\ Universidad de La Frontera \\ Temuco, Chile \\ ccares@ufro.cl
}

\author{
Xavier Franch \\ Departament de Llenguatges i Sistemes Informàtics \\ Universitat Politècnica de Catalunya \\ Barcelona, Spain \\ franch@1si.upc.edu
}

\section{Category: Technical solution}

\begin{abstract}
The mobile office devices market is currently growing, mainly due to the descending cost of wireless technology as well as the high diversity of functions and features covered. Diversity and proliferation become a hard problem when a person or organization aims at selecting the appropriate device for their particular needs. We propose here a framework for producing device recommendations based on personal or business needs. The framework is articulated through an architecture that includes subsystems for data extraction, recommendation and personalization. All these subsystems operate upon a goaloriented knowledge base whose presentation is the subject of this paper. Our approach is built upon three independent models: a market model, which contains descriptions of the current devices offered in the marketplace; a domain model, which states the needs of the person or organization; a mediator model, which describes the types of devices available. We show the actors and processes around these models. Last, we present a prototype that acts as proof-of-concept of the recommender system.
\end{abstract}

Keywords: component selection; goal-oriented modeling; $i^{*}$.

\section{INTRODUCTION}

The mobile office devices (MODs) market is part of the growing mobile-commerce tendency present worldwide [1-5]. Moreover this market has been called a dominant mobile market ecosystem which basically means that it is able to produce structural changes such as high concentration of activity around integrating the mobile chain value [6]

Mobile commerce implies a diversity of actors and mobile equipments that have a relevant role inside their chain value [7, 8]. MODs exhibit many features and are available in a diversity of device types (Pocket PC, Smart Phones, PDA, etc.). The proliferation of MODs adds complexity to the mobile technology adoption process $[9,10]$. Moreover, MOD functionalities (such as web/wap browsers, voice recorder, mp3 player, GPS, video recorder, e-book reader, meeting scheduler, contact list, etc.) evolve rapidly and become mixed [11] as wireless technology does [12]. Thus, it is not easy to trace a clear boundary among the different available MOD types.

These facts configure a complex context where MOD selection processes take place. Consequently, to help MOD users, several web sites with information about MOD have emerged. In spite of the amount of information they may offer, all of them focus on low-level details of technical specifications, rather than on business goals or user requirements. Therefore, we find a gap among the desires and needs of potential MOD customers and the information available in these web sites, making difficult to carry out an informed selection.

A way to improve this state of the art is to consider the adequacy of classical COTS selection methods. Although some of them recognize the intentional point of view of selection processes (e.g. PORE [13] and CARE [14]) they can be hardly conceived in the MOD selection context, due to the abovementioned characteristics of diversity and proliferation. More adequate is the approach of Rolland et al. [15] that uses concepts such as intentionality, goals and strategies. Among the advantages of using goals we mention: goals can be expressed at different levels of abstraction and organizational levels; they allow covering functional and non-functional concerns; they are less volatile than specific functionalities; and they allow modelling and reasoning about different organizational and technical alternatives [16]. In [17] the idea of goal-oriented matching is further developed and can be used as a basis of goal-based selection processes. To sum up, we have not found any specific approach addressing the MOD selection problem, but we have found enough work on goal-oriented selection to be used as a basis for dealing with diversity and proliferation.

In this paper, we propose a conceptual framework for improving the effectiveness of MOD selection processes. In Section II we present the whole three-model selection framework (3MSF). We propose (Section III) to represent separately the domain where the selection takes place and the market that offers MOD, with an additional third model to communicate them, the mediator model. We propose to use $i^{*}$ (eye-star) [18] as modelling language into which we translate the 3MSF (Sections IV and V). This allows focusing on the goals pursued by the person or organization that makes the selection, and also to describe the functionalities and features of MOD in a highly abstract way. In Section VI we show how predicative logic and, specifically the Prolog programming language, are adequate to implement the $3 \mathrm{MSF}$ approach and become an effective support to decision-making in MOD selection. Section VII provides the conclusion and future work. 


\section{THE THREE-MODEL SELECTION FRAMEWORK}

To approach the research challenge of selecting MOD technologies, we are envisaging a framework that combines several processes around a component-based architecture (see Fig. 1). The main processes are:

- Data extraction. We need to provide means for automatic data extraction and classification from the semi-structured information that MOD providers make public about their offerings. Data mining techniques [19] will be the cornerstone of this process.

- Requirements gathering and profiling. The needs of particular consumers, and groups of consumers, will be elicited and put together. Classical requirements engineering techniques for elicitation, negotiation, etc. [20], may be applied in this process.

- Recommendation. The core of the framework, reconciles needs and market offering. Recommender systems [21] provide the needed technology for driving this process.

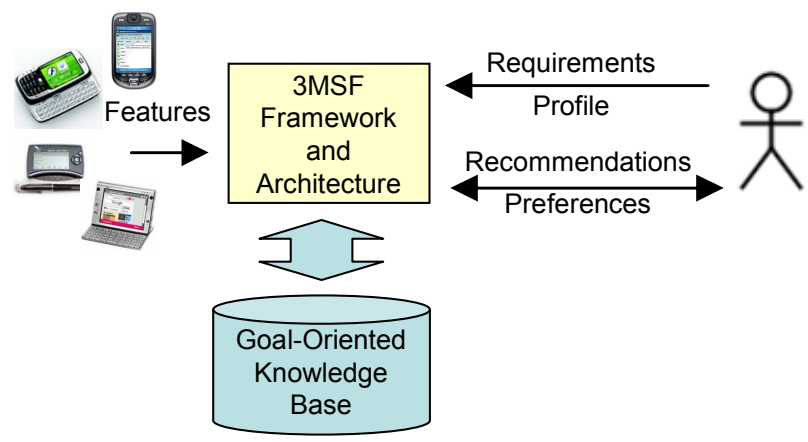

Figure 1. The 3MSF Framework and Architecture

These processes are interconnected by a knowledge base that keeps all the information together. In our current state of research, we have focused on the design of such a knowledge base that will be explained in the rest of the paper, whilst the rest of the framework will be addressed in future work.

\section{THE 3MSF GOAL-ORIENTED KNOWLEDGE BASE}

In a MOD selection context, like in any other market business, we have two clearly identified actors: the customer, who demands some solution, and the industry, that offers products. These actors have different contexts and maybe a dissimilar conceptual framework. For example, while customers talk about face-to-face meeting support, on-line stock checking, price negotiation, etc., industry talks about memory size, bandwidth, web browsers, GPS and resolution. In our first steps towards a framework for MOD selection, we initially tried to join these two points of view into a single solution using an $i^{*}$-based goal-oriented approach, but the result was not satisfactory enough. In spite of this, we found the use of $i^{*}$ valuable as a powerful conceptual tool, therefore we decided to persist with $i *$ to keep the goal-oriented value, but modeling domain and market information separately. To reconcile both contexts we introduced a third participant, the mediator model, which has general information that allows matching the domain model with the market model. As a result:

- The design (or re-design) business process model is done independently from the market model and, even by different teams.

- For the domain model, we can make use of the classical knowledge about goal-oriented modelling of organizations and requirements. Furthermore, we can reuse domain models in different MOD selection processes over time.

- The mediator model helps in dealing with the diversity problem, since it describes the types of devices in a consistent way.

- The market model helps in dealing with proliferation, since it describes the functionalities and features of available MOD in a consistent way.

- Furthermore, in the last two cases, models do not change in time, just grow, which makes their maintenance easier. In other words, existing devices seldom require any specific update because their features and functions do not change over time. Concerning types of devices, there may be new or existing ones can be extended, e.g. considering that a type covers new functions. This situation means also an extension, not an update.

In order to illustrate these three components, in Fig. 2 we show a diagram which includes an example of the knowledge embraced in the models and the interactions among them. Note that the domain and market models do not interact directly.

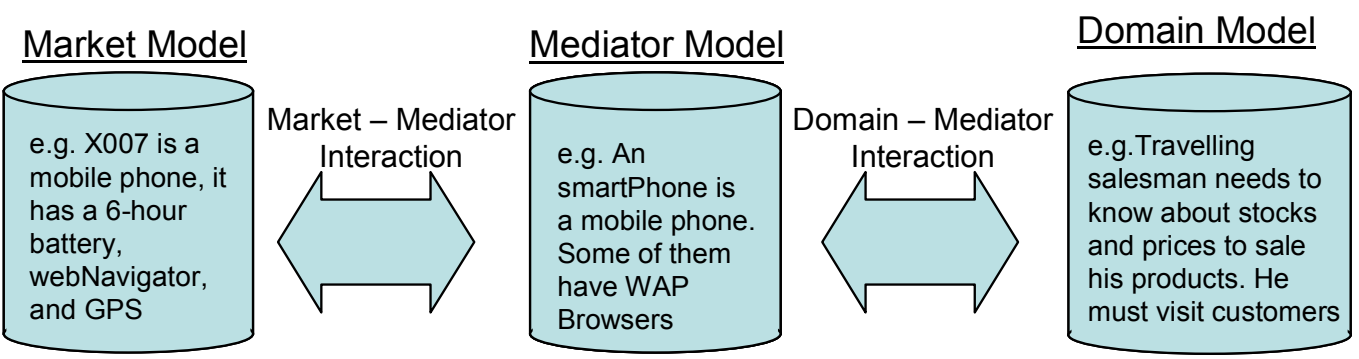

Figure 2. The 3MSF Knowledge Base: general view and some examples 


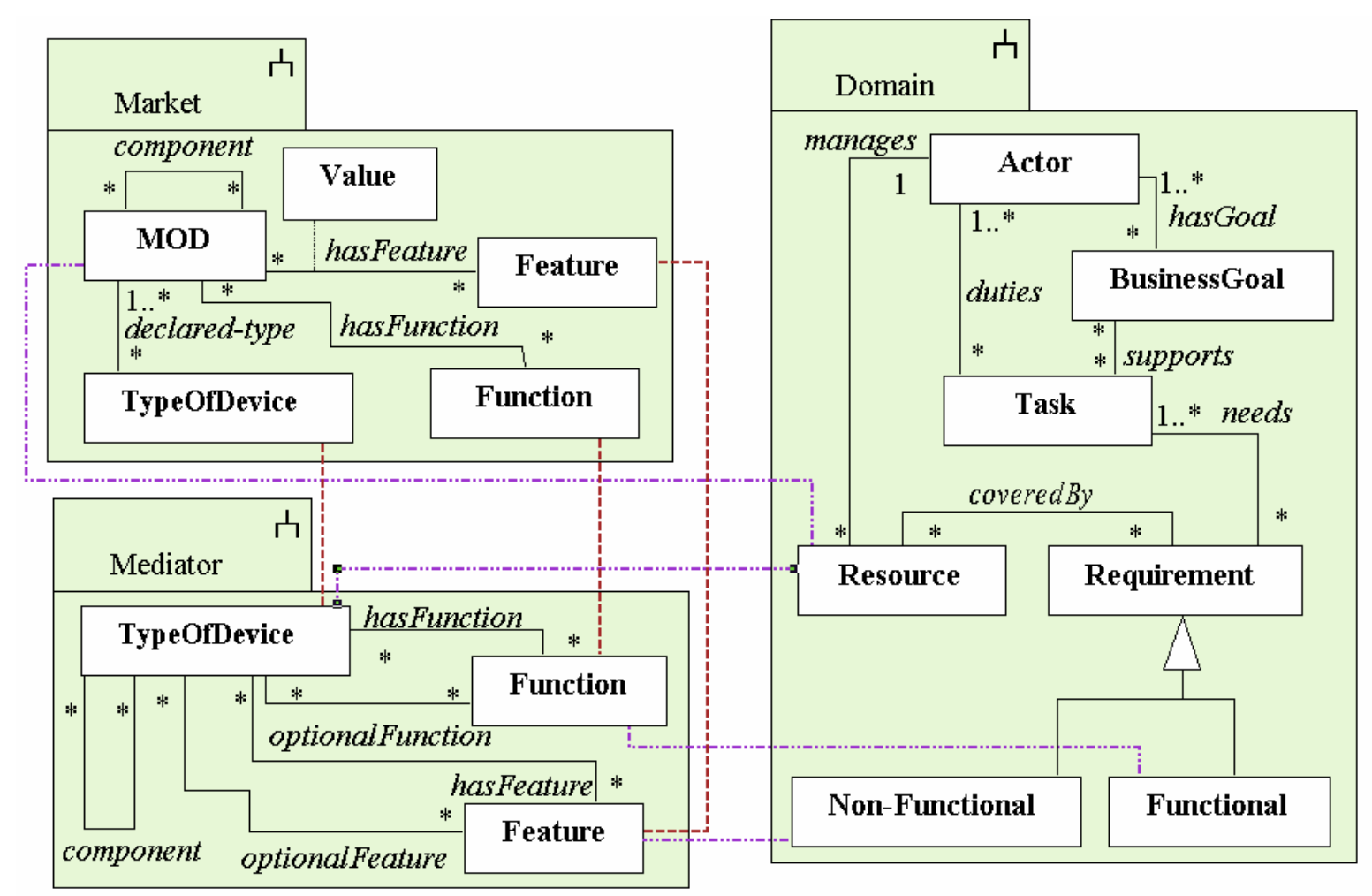

Figure 3. UML Class Diagrams for the Models in the 3MSF Framework

\section{A. Structure of the Models}

There are three differentiated diagrams (see Fig. 3):

- The Domain model declares which actors are interested in the selection process, which are their goals and which tasks do they support. Actors are capable of performing (or obliged to perform) tasks. Tasks state requirements that may be functional or non-functional. These requirements are the ones that generate the need for resources helping the actors to carry out the tasks.

- The Market model specifies which functions cover a particular device, which values do its features take (association class Value) and which are its components. MOD suppliers declare them as belonging to one or more device types (which may be not true, see below).

- The Mediator model records which functions and features (either mandatory or optional) apply to each type of device. Types may have other types as components.

It is worth to remark that in the market model, a particular MOD is associated to one or more types of devices following the beliefs of the supplier (association declared-type) but, on the other hand, the mediator may classify it as belonging to another type or types. Also, we do not force the MOD to satisfy the functions, or establish the value of the resources, of all the functions and resources of its types. In other words, a MOD may cover functions and present features that do not belong to any of its types, and/or some of the functions and features of its types may not be realized in the MOD, and/or there may be a mismatch about what the supplier thinks a kind of component is and what the mediator establishes. These conflicts and mismatches reflect the real situation in the MOD market and aligns with the statement given in the introduction about uncertainness of barriers among types; of course, for our proposal to be effective, this situation should be the exception and not the rule. During the matching process, the user decides if mediator's beliefs override suppliers' information or the other way round, and hence if conflicting information is left or reconciled.

The concepts that appear in the three models are not independent (see dotted lines in Fig. 3). The similarities among the market and the mediator are clear just considering the names. Concerning the domain model, the resources that cover requirements are bound to MOD from the market but also to types of devices, functional requirements are bound to functions and non-functional requirements to features. The dynamic aspects of $3 \mathrm{MSF}$ will show how these semantic equivalences are used.

\section{B. Interaction among the Models}

Fig. 4 shows the general overview of the 3MSF selection process as a UML use case diagram with 3 included use cases and the activity diagrams of each of these 3 use cases; the included use cases can be intertwined as required for particular selection processes. The activity diagrams show optional activities to illustrate the process customization to user needs. 


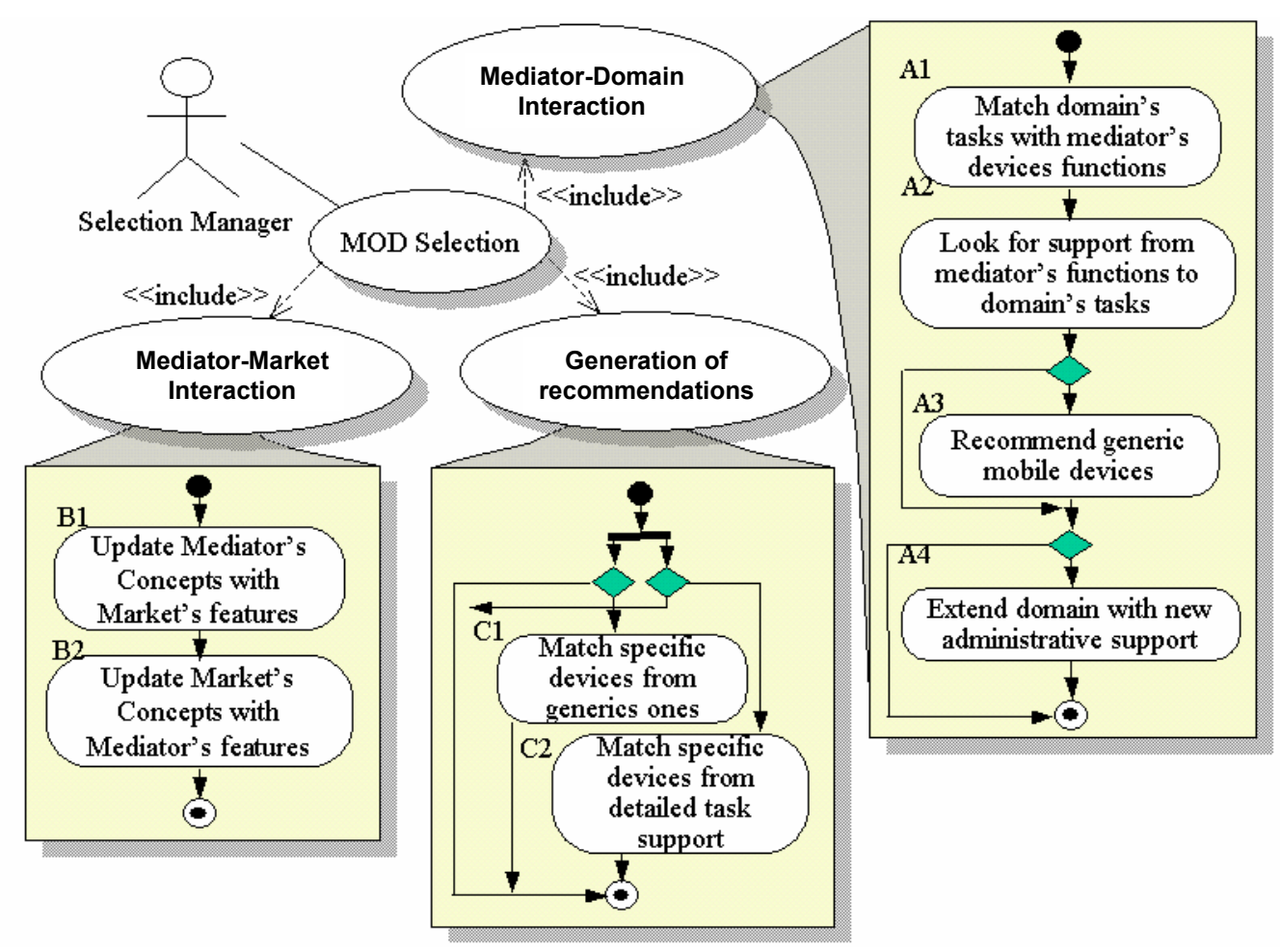

Figure 4. The Selection Process in the 3MSF Framework

In our framework, we model MOD selection processes along three different interactions: (A) the Mediator-Domain interaction; (B) the Mediator-Market interaction; (C) the Recommendation Process itself. The activities inside each interaction are shown in more detail in Table I. We also indicate which activities can be fully automated (auto label) and which ones are user-assisted (assisted label).

The interactions that involve the domain model, i.e. (A) and (C), are the real core of the selection process. The domain model aim is to recommend which device types are the most appropriate for the user. These device types are incorporated as resources in the domain model together with their rationale (e.g., consequences of incorporating these types of devices in the domain). In this process, we are more interested in types of devices than in the devices themselves. In this interaction, correspondences among both models (e.g., how customer needs are mapped onto technological concerns) are established by user assistance and some automatic name matching between elements of both models.

The mediator-market interaction completes information about devices and types (a cross-checking among the mediator and the marketplace). More precisely, the mediator adjusts its model with new information about tendencies from the marketplace, either by defining new types of devices or by adding new functions or features to existing ones. Conversely, existing MODs can be investigated for detecting, and adding if necessary, functions and features that should be included in the market model according to the types of devices the MOD belongs to. This interaction ensures the timely evolution of this highly dynamic market. During the matching process, the user determines if mediator's beliefs override suppliers' ones or if conflicting information is left or reconciled.

Once the conceptual framework has been unified, the Recommendation Process takes place by matching required features against types of devices and, then, adding information about the support of particular mobile devices to domain's tasks (and therefore to domain's goals). The approach allows first identifying a type of device and then a set of individual devices. Both processes can be automated.

\section{THE $I^{*}$ FRAMEWORK: AN INTRODUCTION}

The $i^{*}$ framework [18] proposes the use of two models, each corresponding to a different abstraction level: a Strategic Dependency (SD) model represents the intentional level and the Strategic Rationale (SR) model represents the rational level.

A SD model consists of a set of nodes that represent actors and a set of dependencies that represent the relationships among them, expressing that an actor (depender) depends on some other (dependee) in order to obtain some objective (dependum). The dependum is an intentional element that can be a resource, task, goal or softgoal. It is possible to define the importance (strength) of the dependency for each of the involved actors using three categories: open, committed and critical.

A SR model allows visualizing the intentional elements into the boundary of an actor in order to refine the SD model with reasoning capabilities. The dependencies of the SD model are linked to intentional elements inside the actor boundary. The elements inside the SR model are decomposed accordingly to two types of links: 
TABLE I.

3MSF PROCESS DESCRIPTION

\begin{tabular}{|c|c|c|}
\hline Activity & Description & Example \\
\hline \multicolumn{3}{|c|}{ Mediator-Domain Interaction } \\
\hline $\begin{array}{l}\text { A1 } \\
\text { auto }\end{array}$ & $\begin{array}{l}\text { Correspondences among domain concepts and device types } \\
\text { capabilities are identified, e.g. by comparing names. }\end{array}$ & $\begin{array}{l}\text { The meeting scheduler task from the travelling-salesman domain, matches with } \\
\text { the meeting scheduler function in the } P D A \text { device type, from Mediator. }\end{array}$ \\
\hline $\begin{array}{c}\mathrm{A} 2 \\
\text { assisted }\end{array}$ & $\begin{array}{l}\text { Positive contributions from types of devices capabilities to } \\
\text { domain concepts are proposed to the user based on past } \\
\text { experiences. New contributions (either positive or negative) are } \\
\text { established. }\end{array}$ & $\begin{array}{l}\text { From past experiences, it is established that a GPS Navigator function, from } \\
\text { Mediator, could support positively the task drive the car to the customer place } \\
\text { from the domain of the current selection process. This task is a variation from the } \\
\text { past drive the car to the University task referenced in a previous process. }\end{array}$ \\
\hline $\begin{array}{l}\text { A3 } \\
\text { auto }\end{array}$ & $\begin{array}{l}\text { Devices are numerically ranked using the results of the previous } \\
\text { activities and then qualitatively assessed. A recommended } \\
\text { device could be incorporated as a resource in the domain } \\
\text { model }\end{array}$ & $\begin{array}{l}\text { A } P D A \text { can provide the } 92 \% \text { of your supported set of administrative tasks. This } \\
\text { device is highly recommended. Therefore, it is included in the current solution. }\end{array}$ \\
\hline $\begin{array}{c}\text { A4 } \\
\text { assisted }\end{array}$ & $\begin{array}{l}\text { Functions that are supported by the recommended types of } \\
\text { devices can be incorporated as tasks in the domain model } \\
\text { instead of the type itself. }\end{array}$ & $\begin{array}{l}\text { A set of specific functions as Web browser, GPS Navigator and others are } \\
\text { proposed to extend the domain model. The user may choose to accept all, some } \\
\text { or none. }\end{array}$ \\
\hline \multicolumn{3}{|c|}{ Mediator-Market Interaction } \\
\hline $\begin{array}{c}\text { B1 } \\
\text { assisted }\end{array}$ & $\begin{array}{l}\text { The current state of the market is analysed to discover new } \\
\text { functions, features, devices and types of devices, and the } \\
\text { mediator model is updated with these new findings. }\end{array}$ & $\begin{array}{l}\text { There is a new type of device called smart phone. It always has a phone } \\
\text { communicator function. Due to last technological advances, the battery time } \\
\text { feature must be split into the talk-time battery time feature and stand-by battery } \\
\text { time feature. }\end{array}$ \\
\hline $\begin{array}{c}\text { B2 } \\
\text { assisted }\end{array}$ & $\begin{array}{l}\text { The resulting state of the mediator model is used to update the } \\
\text { information about the market model. New categorizations for } \\
\text { current products in market are recommended. }\end{array}$ & $\begin{array}{l}\text { The Pocket PC can be considered a smart phone } X 007 \text { too. Information about the } \\
\text { talk-time battery time has not been found in the } X 007 \text { model. It is necessary to } \\
\text { specify this feature or to state that is an optional feature on smart phones }\end{array}$ \\
\hline \multicolumn{3}{|c|}{ Recommendation Process } \\
\hline C1 & $\begin{array}{l}\text { When there are generic devices in the domain model (i.e., } \\
\text { device recommendations that were accepted during A3) their } \\
\text { features and functions are searched in the market model and the } \\
\text { specific devices evaluated. }\end{array}$ & $\begin{array}{l}\text { A } P D A \text { has been recommended and incorporated to the domain model. There are } \\
45 \text { PDA's in the database; there are } 3 \text { in this set that achieve a maximum } \\
\text { administrative support and therefore presented to the user. }\end{array}$ \\
\hline $\begin{array}{l}\mathrm{C} 2 \\
\text { auto }\end{array}$ & $\begin{array}{l}\text { When there are detailed task supports (i.e. detailed task } \\
\text { extensions were accepted in A4), all the devices in the market } \\
\text { model are evaluated matching directly features and functions } \\
\text { from market and domain. }\end{array}$ & $\begin{array}{l}\text { There is not any generic device in the domain, but the detailed task support } \\
\text { analysis shows that X007 smart phone and Y008 PDA are very recommended } \\
\text { devices. There are another } 8 \text { devices could be examined in depth since they cover } \\
\text { the business goals up to an acceptable extent. }\end{array}$ \\
\hline
\end{tabular}

- Means-end links establish that one or more intentional elements are the means that contribute to the achievement of an end. The "end" can be a goal, task, resource, or softgoal, whereas the "means" is usually a task. There is a relation OR when there are many means, which indicate the different ways to obtain the end. The possible relationships are: Goal-Task, Resource-Task, Task-Task, Softgoal-Task, SoftgoalSoftgoal and Goal-Goal (following usual conventions, the left-hand side of each pair represents the end and the right-hand side, the means). In Means-end links with a softgoal as end it is possible to specify if the contribution of the means towards the end is negative or positive.

- Task-decomposition links state the decomposition of a task into different intentional elements. There is a relation AND when a task is decomposed into more than one intentional element. It is also possible to define constraints to refine this relationship. The importance of the intentional element in the accomplishment of the task can also be marked in the same way that in dependencies of a SD model.

Actors can be specialized into agents, roles and positions. A position covers roles. The agents represent particular instances of people, machines or software within the organization and they occupy positions (and as a consequence, they play the roles covered by these positions). The actors and their specializations can be decomposed into other actors using the is-part-of relationship.

An example of using the graphical notation is shown in Fig. 5 using the example of a contemporary travelling salesman. On the left-hand side, we show the SR model of a travelling salesman and the hierarchical relationships among their internal intentional elements. On the right-hand side, we show the strategy dependencies between him/her and a customer. 


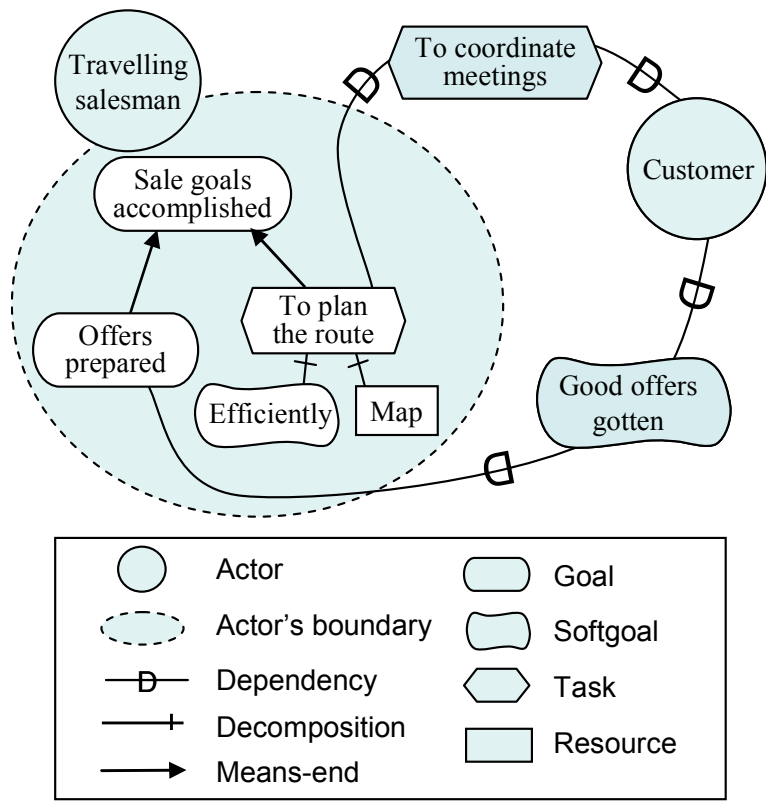

Figure 5. The i* Framework: an Example

\section{MODELLING THE 3MSF USING $I^{*}$}

The $i^{*}$ framework provides a goal-oriented modelling language very well-suited for our purposes. It allows modelling actors' networks in complex socio-technical scenarios. In this section we focus on using the $i^{*}$ constructs presented in the previous section in each 3 MSF model. Thus, the aim of the section is to make use as needed of the standard concepts of $i^{*}$.

In order to make our approach more usable, in the domain model we do not provide any specific guideline. Thus, we are accepting any external modelling approach; as an additional benefit, this freedom allows reusing existing $i *$ domain models, specifically in the form of SR diagrams. The usual case for these diagrams will be to have some tasks in the lower levels and precisely these are the elements that may be used to make explicit the interaction mediator-domain, because the functionalities implied by the tasks are the ones that could be supported by the mobile technology. Other elements like goals and softgoals appear as usual but they do not play such a crucial goal for the recommendation process itself (i.e. the matching process) because at the end of the domain analysis they should have been decomposed into tasks. In particular, the recommended types of devices could take the form of resources if they have been incorporated into the domain model. However, goals and softgoals are useful when we consider goal-oriented analysis, e.g. when we want to find out the consequences of having (or not) a task covered by some MOD.

In the case of the mediator model, we observe that specific technological functions, e.g. Web Browser or MP3 Player, can be associated to administrative functions, so we can model them as roles. Different types of devices may cover one or more of these roles, yielding to a more generic abstraction level of devices, e.g. generic concepts such as PDA or Smart Phone. According to the $i *$ rationale, this means that types of devices are better modelled by positions covering these roles.
Concerning the market model, we represent specific devices using the agent construct since an agent represents a specific individual [18]. Thus, the John Doe's X700 PDA with extended $128 \mathrm{Mb}$ is an agent. If we think that a specific product, e.g. $X 700 P D A$, is a PDA, then we propose that a specific MOD can be represented as a position (not as an agent). Both types of positions (types of devices and devices themselves) are connected using the is_a relationship. Following this idea we represent separated components modelled also as positions. To represent that a component is integrated in a specific device we use the is_part_of relationship. With the above conventions we can say, for example, that your $P D A$ occupies the position of $X 700$, and it is a (is_a) $P D A$, it also covers the generic roles of a PDA. The case of the memory extension is one of the two situations that the semantics of $i^{*}$ does not allow to represent and it needs some extension.

We have already suggested that features also play an important part in 3MSF. The only way to represent (general) features in $i^{*}$ is by means of softgoals that allow to specify properties like fast, cheap, reliable, i.e. non-functional requirements. This may work on the domain model, but not in the others. In them, we need additional constructs to represent precise features like memory size (with value 512) or screen resolution (with value high). So we propose an attribute construct that can be associated to any intentional element, even to actors. We represent this new construct using trapezes with the name of the attribute inside and, optionally, the value of the attribute, when it is known.

Besides, we have mentioned that, in the mediator model, device types are represented as positions and their functions like other covered roles. However some of these functions could be optional. We need an additional construct to embody the fact that "sometimes the position P covers the role R". This relationship is necessary because we need to represent the fact that a MOD of type $\mathrm{X}$, is always an $\mathrm{X}$, despite it has not the function $\mathrm{Y}$ which is part of the usual offering for type $\mathrm{X}$, e.g. the device $d$ is a mobile phone although $d$ does not have a contact list. We call this construct sometimes covers or simply st-covers, therefore we can say in the example that the device of type mobile phone sometimes covers the contact list function. With these two extensions (that may be easily integrated into the $i^{*}$ metamodel presented at [22]), we have completed the proposal to use $i^{*}$ as modelling language for our 3MSF approach. A summary of $i *$ suggestions to apply 3MSF is shown in Table II.

To illustrate the use of $i *$ in the framework, we present an application example. In Fig. 6 we show a portion of the domain model (left-hand side) and mediator model (in the middle). We suggest a reduced initial mediator model, because each model is dynamic, i.e., it grows with new facts. In the example we show the basic concept of PDA modelled as a position. The model states that a PDA covers functions as Contact List and emailer (roles). Also, a PDA sometimes covers the function of a Web browser. Last it is stated that any specific PDA should have features such as weight and price. Of course, features may have the same name in different roles. 
TABLE II. USE OF I* CONSTRUCTS IN THE THREE-MODEL SELECTION FRAMEWORK (3MSF)

\begin{tabular}{|c|c|c|c|}
\hline Model & Concept & i* construct & Example \\
\hline Domain & Unrestricted use of $i^{*}$ & All constructs & Any $i *$ diagram \\
\hline \multirow{6}{*}{ Mediator } & Device type & Position & PDA \\
\hline & Function & Role & Web browser \\
\hline & Feature & Attribute & Weight \\
\hline & Device type has function & Covers & PDA covers Web browser \\
\hline & Sometimes a device type has function & St-Covers & PDA st-covers GPS Navigator \\
\hline & Type of device has another as component & Is_part_of & Processors are part of PDA \\
\hline \multirow{7}{*}{ Market } & Specific device & Position & PalmOne Zire72 \\
\hline & Device type & Position & PDA \\
\hline & Function & Role & Web browser \\
\hline & Feature & Attribute & Weight:5oz \\
\hline & Specific device corresponds to device type & Is_a & PalmOne Zire 72 is_a PDA \\
\hline & Specific device has a function & Covers & PalmOne Zire 72 covers Web browser \\
\hline & Specific device has another specific device as component & Is_part_of & ARM Intel is_part_of PalmOne Zire 72 \\
\hline
\end{tabular}

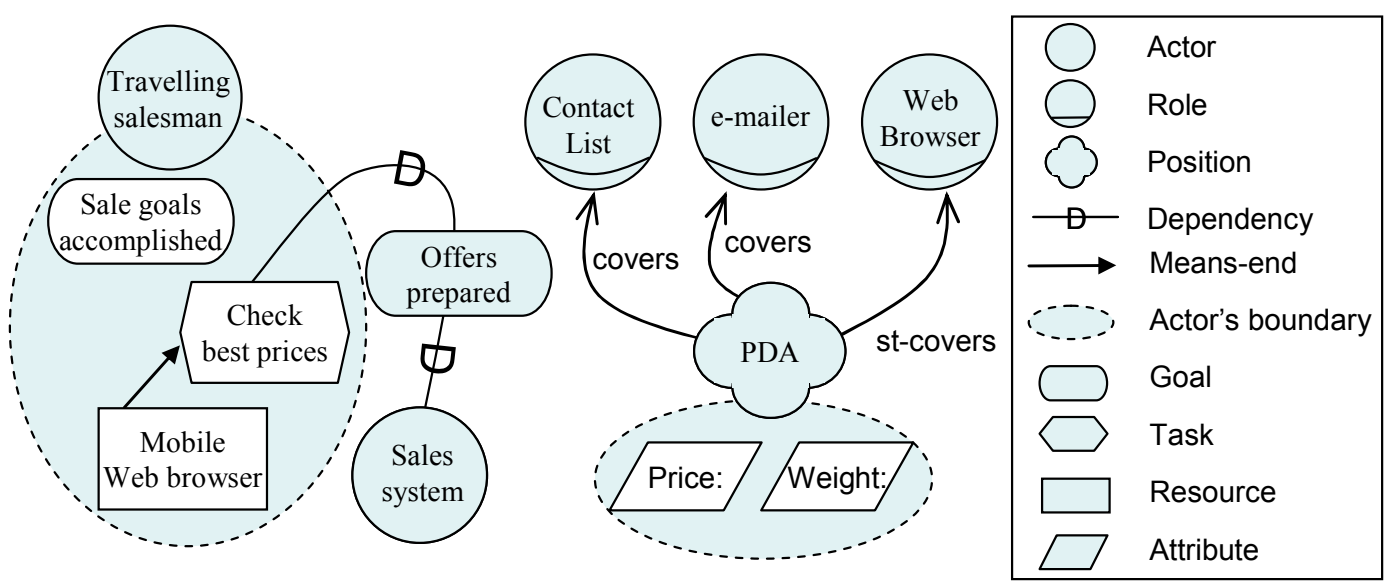

Figure 6. Portions of the Domain Model (left) and Mediator Model (center)

To illustrate the domain model we have chosen a traditional computer science problem as the travelling salesman, but from a different perspective than usual, i.e. with the intention to facilitate his/her work using MOD. The relevant part of the domain model is the rationale inside the travelling salesman boundaries, because it is the place where his/her duties are specified and the possible support of MOD could happen. In general, any task could be candidate for MOD support. In the case of the Fig. 4, it has been identified that the task Check best prices could be supported by a mobile device. However in our complete model other tasks such as check products availability, get products from store, drive the car were also identified.

In the case of market model we built the model following the technical specification from the manufacturer. So, for example, for the device HP iPaq H1945, the manufacturer states that it is a Pocket PC (position), it has a GPS Navigator (position) and the package should include an AC Adapter (resource), among others.
Finally, we expand our representation for 3MSF suggesting the way to implement the activities presented in Fig. 4 using $i *$. Thus we extend Table II with two additional columns, one for expressing the activity in terms of $i^{*}$, and the second to show a specific use in the travelling salesman example. The result is illustrated in Table III.

\section{TOWARDS A KNOWLEDGE-BASED COMPUTER- AIDED DEVICE SELECTION}

A fundamental issue for 3MSF dissemination is to implement some tool support for assisting mobile office technology selection. We present in this section a software system that basically implements the search in the marketplace and that may be considered as the kernel of a future recommender system [21]. The software system requires human interaction to produce a real match of administrative tasks with mobile devices, as specified by the activities of Fig. 4. 


\begin{tabular}{|c|c|c|}
\hline Activity & $i^{*}$ use & $i *$ travelling salesman example \\
\hline \multicolumn{3}{|c|}{ Mediator-Domain Interaction } \\
\hline A1 & Match domain tasks with mediator roles. & There is not initial matching in current models \\
\hline A2 & Ask for contributions from mediator roles to domain tasks & Does a web browser contribute to check best prices? \\
\hline A3 & $\begin{array}{l}\text { The positions that cover the above roles in the mediator model } \\
\text { are evaluated }\end{array}$ & $\begin{array}{l}\text { The PDA position is evaluated because web browser, contact list and e-mailer } \\
\text { have been detected as contributions to some travelling salesman tasks that } \\
\text { appeared previously in other selection processes. }\end{array}$ \\
\hline $\begin{array}{c}\text { A4 } \\
\text { assisted }\end{array}$ & $\begin{array}{l}\text { All the well-ranked positions are suggested as possible selected } \\
\text { devices. All the contributions are suggested to be incorporated } \\
\text { in the domain model. }\end{array}$ & Does the user want to add detected contributions as tasks in the domain model? \\
\hline \multicolumn{3}{|c|}{ Mediator-Market Interaction } \\
\hline B1 & $\begin{array}{c}\text { The market positions are analysed, new is_a relations can be } \\
\text { new device types, new roles can be new functions and new } \\
\text { attributes can be new features. }\end{array}$ & $\begin{array}{l}\text { Do you want to add the Pocket PC as a new type of MOD device? } \\
\text { Do you want to add the weight as a feature for all Pocket PC? }\end{array}$ \\
\hline B2 & $\begin{array}{l}\text { The mediator positions are analysed, new features and roles can } \\
\text { be undeclared attributes in the market model. }\end{array}$ & $\begin{array}{c}\text { Does it make sense to ask for resolution in HP iPaq H1945? Has Pocket Outlook } \\
\text { (the e-mailer) Multiple Windows? Has HP iPaq H1945 a Contact List? }\end{array}$ \\
\hline \multicolumn{3}{|c|}{ Recommendation Process } \\
\hline C1 & $\begin{array}{l}\text { Match positions that represent the devices in mediator model } \\
\text { with positions that have the is_a relationship with the same } \\
\text { name positions in domain model. }\end{array}$ & $\begin{array}{l}\text { The HP iPaq H1945 is a PDA in the market, and a PDA is recommended in the } \\
\text { selection, so this PDA is evaluated for the domain model, trying to match roles } \\
\text { with tasks and evaluating features. }\end{array}$ \\
\hline C2 & $\begin{array}{l}\text { Terminal tasks from domain model match with roles from } \\
\text { market. The positions that cover these roles are candidates to be } \\
\text { the specific devices to be recommended. }\end{array}$ & $\begin{array}{l}\text { A web browser, e-mailer, contact list and calendar are functions that support } \\
\text { travelling salesman. These roles are covered by HP iPaq H1945, so this device } \\
\text { can be recommended. }\end{array}$ \\
\hline
\end{tabular}

The architecture of the system is based on 3MSF and predicative logic. Predicative logic is adequate for symbolic pattern matching and deduction procedures, so we have proposed a knowledge-based system to show that $3 \mathrm{MSF}$ is a feasible approach to the problem of mobile office technology selection.

The interactions between the system and its environment are done through $i^{*}$, which means that the system takes $i^{*}$ models from the environment as input and returns $i^{*}$ models as output. We represent these models textually using predicative logic due to the reasoning ability of this formalism. The name of $i^{*}$ constructs act as predicate names, which makes the translation process easy. In fact, if we agree about the parameter ordering in the predicates representation of $i *$ 's relationships (dependencies, covers, is-a, is-part-of, contributions, etc.), then we have a decidable and simple procedure to transform $i *$ diagrams to predicates, and also, we can easily take back these predicates to build $i^{*}$ diagrams. So, for example we have used some of the following Prolog facts: position(pocketPC), resource(acAdapter), attribute(weight), covers(hpipaqH1945,mp3player), stCovers(pda,webBrowser).

In our prototype we have used the Prolog programming language that takes logic predicative sentences as knowledge, in the case of simple facts, and as rules, in the case of consequences representations, so for a predicative representation it is very suitable. Specifically we have used WSI-Prolog [23, 24] which is under the GNU public license and has more than 15 years of development. It has language interfaces to $\mathrm{C}$ and $\mathrm{C}++$, and besides, there is a CGI package that allows having an interface to HTTP, thus it is possible to offer some MOD selection web service selection with this technology. The prototype has a variable size depending on the predicates inside; for example the mediator model with 15 positions and 92 roles grows up to 350 predicates, which is less than $3 \mathrm{~Kb}$ in memory storage. Any device use less than this, so, if we reach a number such as 1000 devices (or 1024) we will have $3 \mathrm{Mb}$ on disk storage with market information.

The implementation has been done in two stages, first the knowledge base management construction, and second the selection process itself. For the knowledge base management, we have used different Prolog files that contain the representation of each model, which has been taken directly from its predicative form. Second the selection process implementation, which keeps the dynamic 3MSF proposal to implement the selection process (see Fig. 4) focusing on the generic recommendations process. Thus, we have got an initial Prolog prototype that implements the knowledge database and the generic selection. 


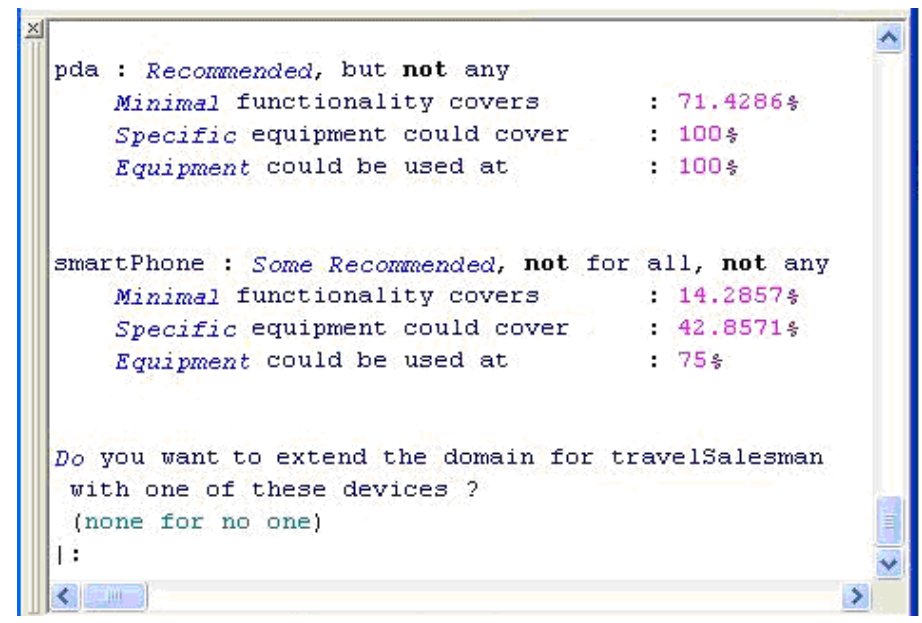

Figure 7. Screenshot: a Recommendation for Generic Devices

In Fig. 7 we show a screenshot where it is possible to see a generic device evaluation corresponding to activity A3 (see Fig. 4 and Table 2). This example says that a PDA is recommended but not any PDA, because there are st-covers relationships to some of the required functions, this means that just some PDA have these special functions that are required by the travelling salesman. Also it is said that the generic concept of PDA covers the $71 \%$ of requirements but a specific suitable PDA could cover all of them. In the case of smart phone it is said that, even in the better cases, there are over the $50 \%$ of requirements that won't be satisfied and, besides, there are a $25 \%$ of basic functions provided by this type of device that are not required.

\section{CONCLUSIONS AND FUTURE WORK}

We have proposed framework called 3MSF for mobile office devices selection that is based on a goal-oriented knowledge base. It illustrates that the goal-oriented approach is effective to keep the focus on the business goals during the selection process. We have approached a solution using separated models for market and domain. Besides we avoid high combinatorial interaction between these models by proposing a mediator model which has abstract knowledge about devices and their uses. The framework is expressed using $i^{*}$ which allows modeling directly complex organizational needs from the mobile office context. We have also proposed a simple way to put these $i^{*}$ models into a predicative representation as a proof-of-concept of the framework. We have shown that this transformation allows getting $i *$ models from a Prolog prototype that may include both generic and specific recommendations for mobile office devices. The approach exhibits the following properties:

- The design (or re-design) of business process models is done independently from the market model and, even possibly, by different teams, which facilitates modelling and reusing.

- It is possible to use classical knowledge about goaloriented modelling of organizations and requirements into domain modelling. Furthermore, we can reuse pre- existing $i^{*}$ domain models in different MOD selection processes over time.

- The mediator model helps in dealing with the diversity problem, since it describes the types of devices in a consistent way and thus represents devices from a higher abstraction level. The classifications may be extended considering new functions.

- The market model helps in dealing with proliferation, since it describes the functionalities and features of available MOD in a consistent way and allows add new devices monotonically, i.e. existing devices do not require any specific update because their features and functions do not change over time.

- Using $i^{*}$ allows modelling directly complex organizational needs from the mobile office context. We have also proposed a simple way to put these $i^{*}$ models in a predicative representation and from then a Prolog implementation does not result a complex task

- $\quad$ The approach recognizes and lives with fuzzy frontiers among device types and information incompleteness about device classifications, functions and features. Both issues are reflected in the two types of selections, focusing either on types of devices or in their insights (functions and features), and finally

- It generates the outlined interactions and recommendations oriented to MOD selection based on organization goals.

Although in this work we have focused in the MOD domain, the general architecture and most of the concepts could be thought to be applied to other domains with similar characteristics: existence of a huge marketplace that evolves quickly, where the problem of alignment among users' needs and marketplace offering is a challenge. Extending the $3 \mathrm{MSF}$ framework to the general problem of selection in this context is part of our future work.

Related work is presented in [25], where it is addressed the problem of mobile equipment selection considering the multicriteria decision method. This approach requires a stable knowledge of the application domain and also a clear conceptual framework about devices classifications and functionalities. We see quantitative approaches like this would be a second and complementary step to our proposal.

Since we are still shaping our proposal, we have identified several current limitations that generate further research challenges. Perhaps the most significant one is that we have not distinguished the concepts of fabricant and supplier of devices in the market. Implicitly, we have just one description of each device which means that each device is published just once in the market. The real situation is that besides fabricants such as Nokia or HP we have suppliers that commercialise these devices. Although suppliers could even offer the same set of products, there may be relevant differences among them, for example warranty times, repair services included, special offers for big deals and others that could be critical in decisionmaking. 
Some other minor drawbacks follow. It is implicit that all business tasks have the same priority, which is not necessary true, this could be a problem if there is a small set of exclusive functions that must be satisfied. The way to deal with this problem currently would be not considering secondary tasks in the first mediator-domain interaction, but adding them in a second run, but obviously this is not the best option.

Another topic is the weight of features. In our current version we have not offered the option to set limit values for features neither it is possible to set some level of relevance for them. This could be important for features like price or some mandatory functional or non-functional requirements. Also concerning features, we have not taken into account the possible diversity of measurement units. This is an interesting property because not always the manufacturers use the same measure units.

Besides solving the above limitations, in terms of future work we would like to introduce the concept of profile in domains. For instance, instead of building a whole $i^{*}$ model from the scratch, a new problem of selection could identify mobiles profiles and compose and refine them. Examples of profiles could be: frequently traveller, disabled person, low budget, being technologically conservative, etc.

But of course the most significant challenge as future work is to refine and evolve the whole framework as presented in Section II. Tool-support is crucial for success. Future tool support will be articulated around 3 different axes: data gathering, models' reuse and deductive power. For the last point, we guess that the current Prolog prototype is a good starting point, and we are working now on improving the clauses that implement the deductive power. We have also begun a project to get a web interface, to add data bases, etc. For data gathering, existing techniques based on data mining, text retrieval and semi-structured information processing would aid in the duty of populating the tool with massive real data. For models' reuse, it is basic to be able to suggest correspondences among domain and the other models whilst the matching process during selection takes place. At this respect we may think of using the iStarML interchange format [27], a XML representation of $i^{*}$ models that allows storing $i^{*}$ domain and market models. It enables an automatic translation from domain models to a predicative representation. On the side of tools, some $i^{*}$ tools start to include iStarML as interchange format, and this broads the scope of tools that can be used to implement 3MSF. On the theoretical side we see the challenge of considering new tendencies to manage variability on $i^{*}$-based goal models $[27,28]$ and to consider this variability into the corresponding predicative representation and logic deduction.

\section{ACKNOWLEDGEMENTS}

We want to thank Enric Mayol for his involvement in the early stages of this research. This work has been partially supported by the Spanish project ref. TIN2007-64753.

\section{REFERENCES}

[1] L. Kwei-Jay, "E-commerce technology: back to a prominent future,". IEEE Internet Computing vol. 12, n1, pp. 60-65, 2008.

[2] M. Megna, "Mobile commerce: tapping the unthethered market," Ecommerce-Guide.Com, 2007

[3] G.P. Boretos, "The future of the mobile phone business,". Business Technological Forecasting and Social Change vol. 74, n3, pp. 331-340, 2007.

[4] N. Willing, "Top 10 Emerging Mobile Markets,". Unstrung http://www.unstrung.com/document.asp?doc_id=119630 Last accessed March 2009, March 2007.

[5] Z.K. Shalhoub and S.L. Al-Qasimi, The Diffusion of E-commerce in Developing Economies: A resource-based Approach: Edward Elgar Publishing, 2006.

[6] H. Verkasalo, A Cross-Country Comparison of Mobile Service and Handset Usage. Licentiate of Science Thesis, Helsinki University of Technology. Department of Electrical and Communications Engineering, Helsinki, 2007.

[7] Y.-F. Kuoa, and C.-W Yu, "3G telecommunication operators' challenges and roles: a perspective of mobile commerce value chain.," Technovation vol. 26, pp. 1347-1356, 2006.

[8] G. Camponovo, and Y. Pigneur, "Analyzing the m-business landscape," Annals of Telecommunications vol. 58, pp.1-2, 2003

[9] A.R. Hickey, "Mobile device trends: security, consolidation and more," http://searchmobilecomputing.techtarget.com/news/article/0,289142,sid4 0_gci1186391,00.html Last accessed March 2009, May 2006.

[10] P. Archer, and E. Mitukiewicz, "Scope of mobile web best practices,".World Wide Web Consortium (W3C) http://www. w3.org/TR/2005/WD-mobile-bp-scope-20050901/ Last accessed March 2009, 2005.

[11] R. Miller,. and E. Roche, "Toward bridge building: mapping the landscape of telecommunication tools," Proc. of the 7th International Conference on Human Computer Interaction with Mobile Devices \& Services, Salzburg, Austria, 2005, pp. 207-214.

[12] P. Ballon, "Changing business models for Europe's mobile telecommunications industry: the impact of alternative wireless technologies,". Telematics and Informatics, vol. 4, n3, pp. 192-205, August 2007

[13] N. Maiden, and C. Ncube, "Acquiring requirements for COTS selection," IEEE Software vol.15, n2, pp. 46-56, 1998.

[14] L. Chung, and K. Cooper K, "COTS-aware requirements engineering and software architecting,". Proc. of Software Engineering Research and Practice Conference (SERP'04), Las Vegas, Nevada, USA, 2004.

[15] C. Rolland C, and N. Prakash, "Matching ERP system functionality to customer requirements,". Proc. of the Fifth International Symposium on Requirements Engineering (RE'01), Toronto, Canada, 2001, pp. 66-75.

[16] A.v. Lamsweerde, "Requirements engineering in the year 00: a research perspective," Proc. of the 22nd International Conference on Software Engineering (ICSE'2000), 2000.

[17] X. Franch, "On the lightweight use of goal-oriented models for software package selection," Lecture Notes in Computer Science, vol. 3250 (CAiSE'05) pp. 551-566, 2005.

[18] E. Yu, Modelling Strategic Relationships for Process Reengineering $\mathrm{Ph}$.D. thesis, Department of Computer Science University of Toronto, Toronto, 1995.

[19] J. Han, and M. Kamber, Data Mining: Concepts and Techniques: Morgan Kaufmann, 2001.

[20] I. Sommerville, "Integrated requirements engineering: a tutorial," IEEE Software vol. 22, n1, pp. 16-23, 2005.

[21] G. Adomavicius, and A. Tuzhilin, "Toward the next generation of recommender systems: a survey of the state-of-the-art and possible extensions,". IEEE Transactions on Knowledge and Data Engineering vol.16, n6, pp. 734-749, 2005.

[22] C. Ayala, C. Cares, J.P. Carvallo, G. Grau,M. Haya, G. Salazar, X. Franch, E. Mayol, and C. Quer, "A Comparative analysis of $i^{*}$-based agent-oriented modeling languages," Proc. of the Conf. on Software 
Engineering and Knowledge Engineering (SEKE'05), Taipei, Taiwan, Republic of China, 2005, pp.43-50.

[23] http://www.swi-prolog.org/. Last visit at March-2009.

[24] J. Wielemaker, "An overview of the SWI-Prolog programming environment," The 13th workshop on logic programming environments. WLPE-03, Numbai, India, 2003.

[25] J. Ondrus, T. Bui, and Y. Pigneur, "A multi-actor, multi-criteria approach for technology selection when designing mobile information systems," Working Conference on Mobile Information Systems (MOBIS'05), Leeds, UK, 2005.
[26] C. Cares, X. Franch, A. Perini, and A. Susi, "iStarML: an xml-based model interchange format for $i^{*}, "$ Proc. of the 3rd International $i *$ Workshop, Recife, Brazil, 2008.

[27] L. Penserini, A. Susi, and J. Mylopoulos, "High variability design for software agents: extending tropos," ACM Transactions on Autonomous and Adaptive Systems vol. 2, n4 p.16, November 2007.

[28] S. Liaskos, A. Lapouchnian, Y. Yu, E. Yu, and J. Mylopoulos, "On goalbased variability acquisition and analysis," 14th IEEE International Requirements Engineering Conference (RE'06), Minneapolis/St.Paul, Minnesota, USA, 2006, pp. 79-88. 